\title{
AN UNUSUAL CASE OF HYDATID CYST OF LIVER AND PANCREAS
}

\author{
Rajeev Kumar1 , Anjali2, Anuranjan Dubey33, Gyanendra Narayan Singh ${ }^{4}$, Vijay Shankar Prasad 5 \\ ${ }^{1}$ Associate Professor, Department of Radiodiagnosis, Patna Medical College and Hospital, Patna. \\ 2Postgraduate Resident, Department of Radiodiagnosis, Patna Medical College and Hospital, Patna. \\ ${ }^{3}$ Postgraduate Resident, Department of Orthopaedics, Patna Medical College and Hospital, Patna. \\ 4 Professor and HOD, Department of Radiodiagnosis, Patna Medical College and Hospital, Patna. \\ 5Professor, Department of Radiodiagnosis, Patna Medical College and Hospital, Patna.
}

\section{ABSTRACT}

\section{BACKGROUND}

Hydatid disease is a cyclo-zoonotic parasitic infection caused by Echinococcus granulosus. This disease is usually found in liver and lungs, but no organ of body is immune. Location at unusual sites in the body can have atypical presentations and can pose a diagnostic challenge. A high index of suspicion, radiological investigations as well as serological examination is necessary in establishing the diagnosis of hydatid disease at unusual sites in the body. We report here a case of a 35-year-old female, who presented with abdominal pain and a lump in the epigastric region.

\section{KEYWORDS}

Hydatid Disease, Computed Tomography (CT), Ultrasonography (USG).

HOW TO CITE THIS ARTICLE: Kumar R, Anjali, Dubey A, et al. An unusual case of hydatid cyst of liver and pancreas. J. Evolution Med. Dent. Sci. 2016;5(91):6795-6797, DOI: 10.14260/Jemds/2016/1535

\section{BACKGROUND}

Echinococcosis also referred to as hydatid disease or echinococcal disease is caused by the larval stages of different species of the tapeworm of genus Echinococcus. ${ }^{[1]}$ Hydatid disease is endemic and mostly affects a single organ, commonly the liver and lung. Pancreatic involvement is of rare occurrence with $<1 \%$ incidence. The life cycle of Echinococcus involves two hosts, one definitive carnivore host (dogs, cats and certain wild carnivores) and the other intermediate herbivore host (Sheep, goats, small rodents and other herbivores). Humans act as an accidental intermediate host; infected after ingesting viable oncosphere-containing eggs, which have been shed in the faeces of the definitive host. The ingested ova penetrate the intestinal wall, reach the portal system and from there to the liver where most of them are lodged in the hepatic sinusoids.[2] A few ova may pass through the liver (First filter) and reach the lung (Second filter) and then into the systemic circulation causing hydatid disease in other organs. A possible dissemination through lymphatic channels accounts for cases with hydatid cysts at uncommon sites. ${ }^{[3,4]}$ In $10 \%$ cases, hydatid disease arises in the viscera; mainly in the spleen $(0.9-8 \%)$ and also in kidney, bone, heart muscles and peritoneal cavity $(0.5-5 \%)$. Possible sources of infestation include haematogenous dissemination, local spread via pancreatobiliary ducts and peripancreatic lymphatic invasion.[5] The head of the pancreas is the most frequent location (57\%) followed by the corpus (24\%) than the tail (19\%).

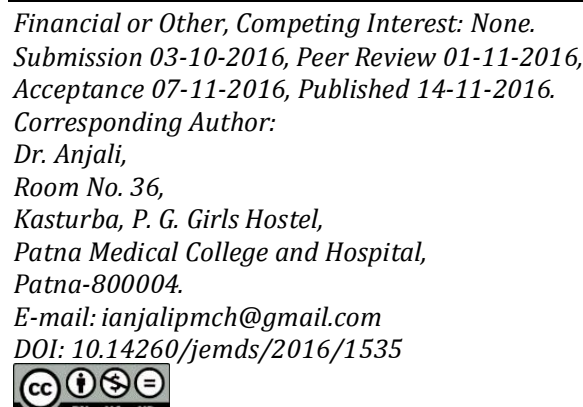

A number of hypotheses regarding the mode of passage of E. granulosus to pancreas have been postulated, the most accepted is the haematogenous dissemination discussed above. The second route involves passage of cystic elements into the biliary system and then to the pancreas. The third route involves passage of cystic elements into lymphatic channels through the intestinal mucosa and then to pancreatic tissue rich in lymphatic network.[6] The fourth route is direct passage of larvae into pancreatic tissue, bypassing the liver via pancreatic veins. Hydatid cysts located in the pancreatic head may cause obstructive jaundice or acute pancreatitis by either exerting external compression on or fistulising into the common bile duct. Less commonly they may lead to cholangitis, duodenal stenosis or duodenal fistula.[7,8] On occasion hydatid cysts in the pancreatic head may remain silent, but be palpable as an epigastric mass lesion. Hydatid cysts in the pancreatic body and tail usually remain asymptomatic until they grow large enough to compress adjacent organs or anatomical structures. ${ }^{[9]}$ USG is a noninvasive, low-cost and sensitive diagnostic technique. Gharbi defined the typical appearance of hydatid cysts in USG.[10] CT is usually successful in delineating cyst size, location, relation with pancreato-biliary system and presence of cysts in other organs. The seropositivity rate is higher in hepatic hydatid cysts than cysts in other organs; however, that seronegativity does not guarantee absence of hydatid disease.

\section{Case Description}

A 34-year-old female patient presented to our Surgical Outpatient Department with epigastric pain since 6 months. She gave no history of vomiting, jaundice, fever or weight loss. On examination, the patient was conscious and well oriented. Abdominal examination revealed mild tenderness. The past medical and surgical histories were unremarkable. Laboratory investigations including complete blood picture, liver function tests and serum amylase were within normal ranges.

\section{Imaging Findings}

Abdominal sonography was performed. Multiple variable sized multiloculated cystic masses in the both lobes of the liver 
were revealed. The largest mass measured $5.5 \times 5.0 \mathrm{~cm}$ in the right lobe of liver with multiple cystic components giving a honeycombed appearance. Some internal membrane-like structures were seen in the centre of the lesion. One of the masses appeared hypoechoic with heterogeneous content within. Another well-defined hypoechoic lesion was seen predominantly in the body of pancreas. This lesion showed areas of peripheral calcification along the anterior and posterior margin. Colour Doppler sonography revealed no significant vascularity within the lesions. Main pancreatic duct was not dilated. Possibilities of hydatid cyst involving liver and pancreas was given on sonography.

CT abdomen was performed to further characterise the lesions. Plain and contrast enhanced CT scan of abdomen showed multiple well-defined hypodense lesions in both lobes of liver. Another lesion was in the pancreas with specks of calcification in its periphery. The lesions did not show contrast enhancement. There was no peripancreatic stranding or any fluid collection. The serological test for hydatid disease (the enzyme-linked immunosorbent assay (ELISA)) was positive. A diagnosis of hydatid disease involving the liver as well as pancreas was made on the basis of imaging and serological findings.

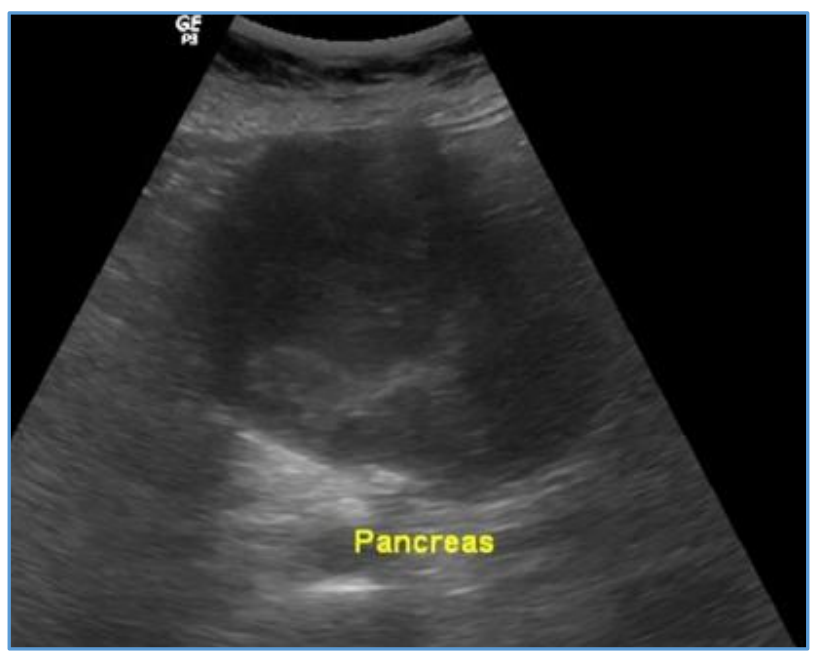

Figure 1. USG shows an Hypoechoic Lesion within Pancreas

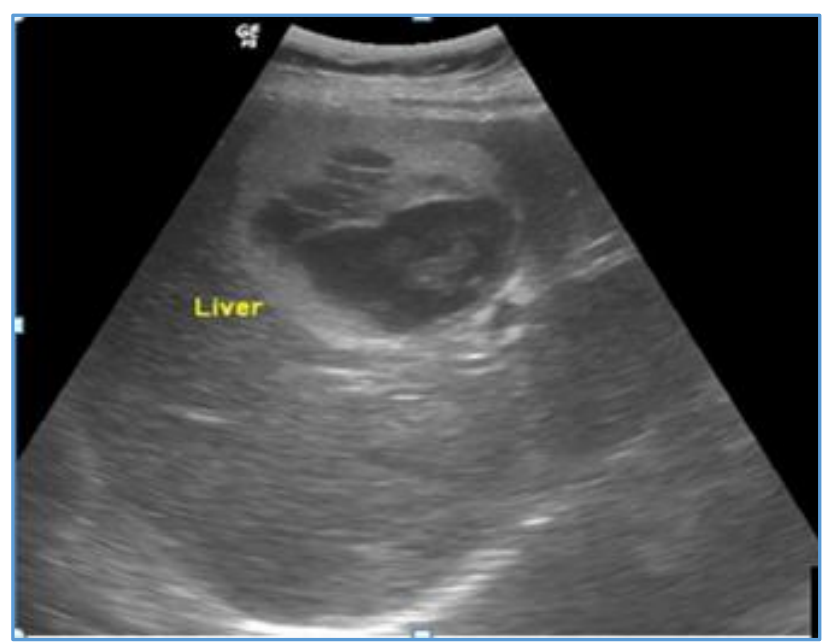

Figure 2. USG shows a Complex Cystic Lesion with Internal Membranes and Septations in the Liver

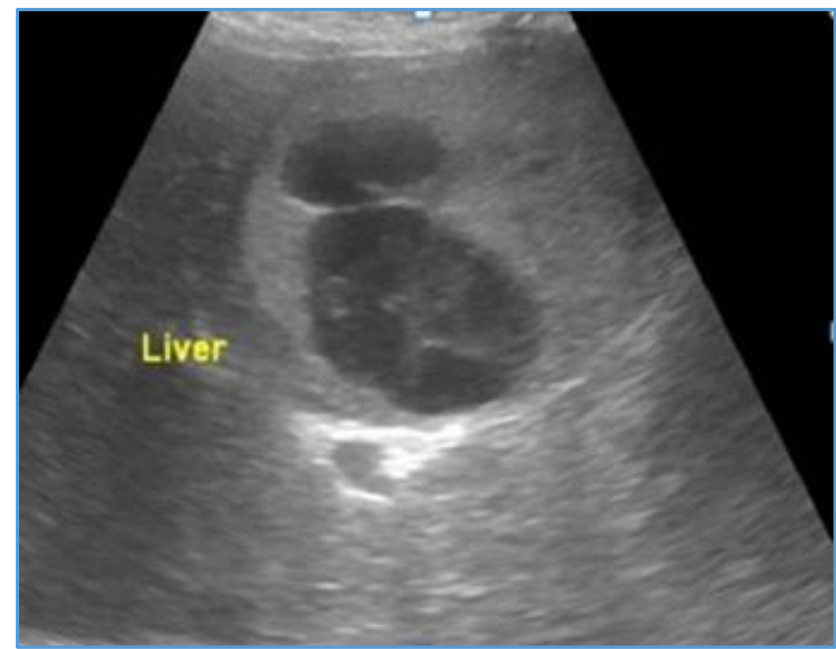

Figure 3. USG shows another Complex Lesion with Daughter Cysts Within

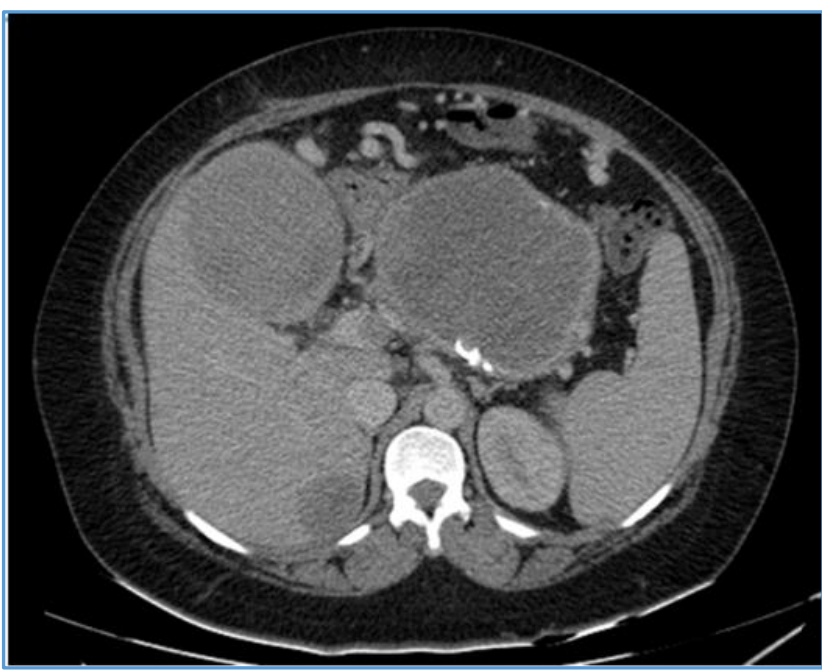

Figure 4. CT shows Cystic Lesions in Pancreas and Liver

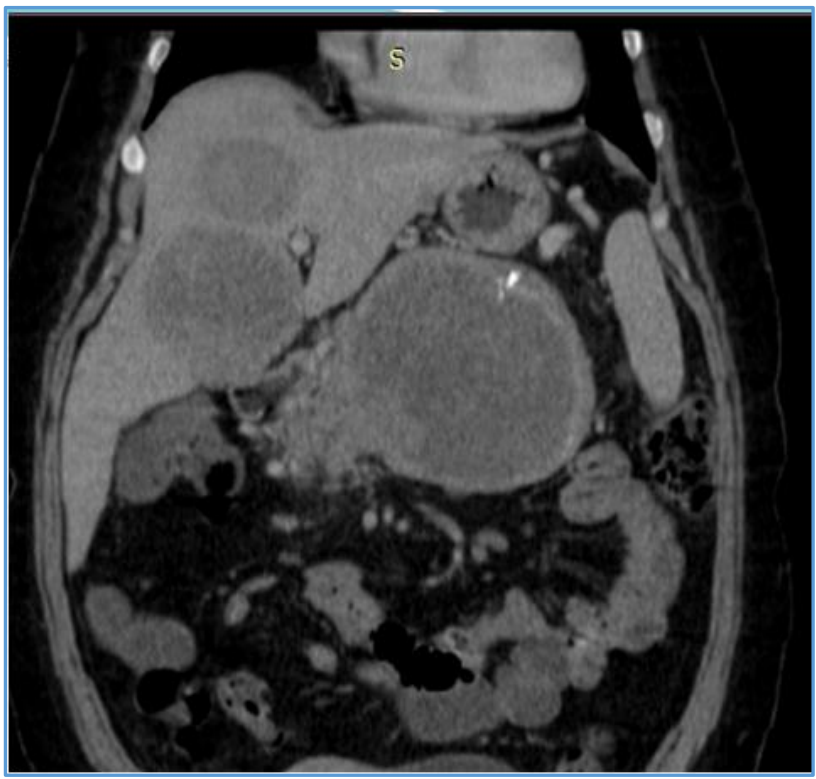

Figure 5. Coronal Reformatted CT shows Multiple Hydatid Cysts in Liver and Pancreas 


\section{DISCUSSION AND CONCLUSION}

Hydatid disease is endemic and most commonly involved organ is the liver. The hydatid cyst of the pancreas is a very rare condition. This case demonstrated involvement of a common (liver) as well as rare site (pancreas). Due to its rarity and similarity with more common pancreatic cystic conditions, diagnosis of pancreatic hydatid cyst may be challenging. As in most cases, hydatid cysts are usually asymptomatic; however, the patients may complain of abdominal pain, vomiting or obstructive jaundice or acute pancreatitis, so we can conclude that in endemic areas any patient presenting with a cystic mass in any tissue or organ should be considered a potential case of the hydatid disease.

\section{REFERENCES}

1. Yuksel M, Demirpolat G, Sever A, et al. Hydatid disease involving some rare locations in the body: a pictorial essay. Korean J Radiol 2007;8(6):531-40.

2. Khuroo MS. Hydatid disease: current status and recent advances. Ann Saudi Med 2002;22(1-2):56-4.

3. Yildirim M, Erkan N, Vardar E. Hydatid cysts with unusual localizations: diagnostic and treatment dilemmas for surgeons. Ann Trop Med Parasitol 2006;100(2):137-42.

4. Marti-Bonmati L, Serrano MF. Complications of hepatic hydatid cysts: ultrasound, computed tomography, and magnetic resonance diagnosis. Gastrointest Radiol 1990;15(2):119-25.
5. Lewall DB, McCorkell SJ. Rupture of echinococcal cysts: diagnosis, classification, and clinical implications. AJR 1986;146(2):391-4.

6. Aydin A, Ersoz G, Tekesin 0, et al. Hydatid acute pancreatitis: a rare complication of hydatid liver disease. Report of two cases. Eur J Gastroenterol Hepatol 1997;9(2):211-4.

7. Bedioui H, Chebbi F, Ayadi S, et al. Primary hydatid cyst of the pancreas: diagnosis and surgical procedures. Report of three cases. Gastroenterol Clin Biol 2008; 32(1Pt1): 102-6.

8. Jai SR, El Hattabi K, Bensardi F, et al. Primary hydatid cyst of the pancreas causing obstructive jaundice. Saudi J Gastroenterol 2007;13(4):191-3.

9. Ousadden A, Elbouhaddouti $\mathrm{H}$, Ibnmajdoub $\mathrm{KH}$, et al. Primary hydatid cyst of the pancreas with a hepatic pedicule compression. Cases J 2009;2:201.

10. Dahniya MH, Hanna RM, Ashebu S, et al. The imaging appearances of hydatid disease at some unusual sites. Br J Radiol 2001;74(879):283-9. 